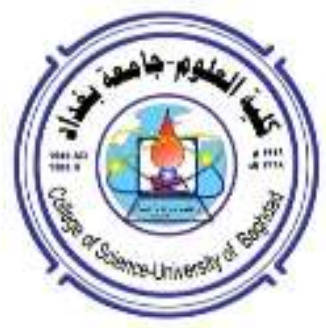

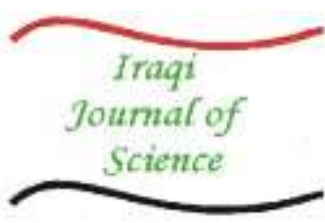

ISSN: 0067-2904

\title{
Large-Coessential and Large-Coclosed Submodules
}

\author{
Amira A. Abduljaleel *, Sahira M. Yaseen \\ Mathematics Department, College of Science, University of Baghdad, Baghdad, Iraq
}

Received:9/3/2021

Accepted: $31 / 5 / 2021$

\begin{abstract}
The goal of this research is to introduce the concepts of Large-coessential submodule and Large-coclosed submodule, for which some properties are also considered. Let $M$ be an R-module and $K, N$ are submodules of $M$ such that $K \leq N \leq M$, then $K$ is said to be Large-coessential submodule, if $\frac{N}{K} \ll_{L} \frac{M}{K}$. A submodule $N$ of $M$ is called Large-coclosed submodule, if $K$ is Large-coessential submodule of $N$ in $M$, for some submodule $K$ of $N$, implies that $K=N$.
\end{abstract}

Keywords: L-small submodule, L-coessential submodule, L-coclosed submodule

المقاسات الجزئية الضد الجوهرية الاساسية والمقاسات الجزئية الضد المغلقة الاساسية

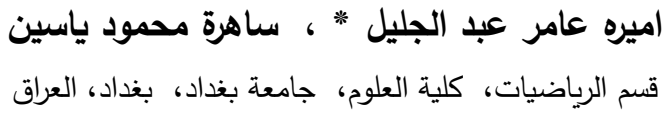

الخلاصة

الغرض من هذا البحث هو تقديم مفاهيم المقاسات الجزئية الضد الجوهرية الاساسية والمقاسات الجزئية

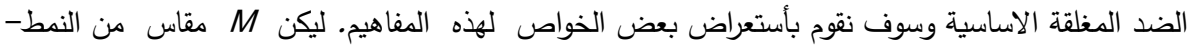

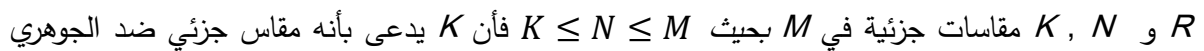

الاساسي , اذا كان

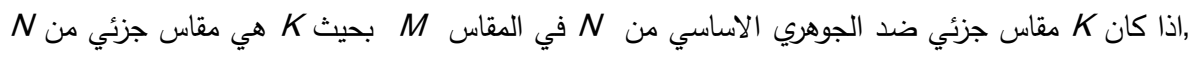

$$
\text { يؤدي الى انه }
$$

\section{Introduction}

Throughout this paper, R will be a commutative ring with identity. A proper submodule $N$ of an R-module $M$ is called small $(N \ll M)$, if for any submodule $K$ of $M$ such that $N+$ $K=M$, implies that $K=M$ [1]. A proper submodule $N$ of an R-module $M$ is called Large (essential) submodule in $M,\left(N \leq_{e} M\right)$, if for every non zero submodule $K$ of $M, N \cap K \neq 0$ [1]. A submodule $N$ of $M$ is called closed in $M$ if it has no proper essential extension in $M$ [2]. For $K \leq N \leq M, K$ is called coessential submodule of $N$ in $M\left(K \leq_{c e} N\right)$ if $\frac{N}{K} \ll \frac{M}{K}$, and $K$ is said to be coclosed in $M$ denoted by $\left(K \leq_{c c} M\right)$, if $K$ has no proper coessential submodule in $M$ [2,3]. In an earlier study [4], the concept of Large-small submodule was introduced, such that a proper submodule $N$ of $M$ is called Large-small ( L-small ) submodule of $M$, denoted by $\left(N \ll_{L} M\right)$. If $N+K=M$, where $K \leq M$, then $K$ is an essential submodule 
of $M\left(K \leq_{e} M\right)$. It is clear that every small submodule of $M$ is L-small submodule of $M$, but the converse is not true. Many authors have been interested in studying different generalizations of coessential and coclosed submodules [5-8]. In this paper, we introduce the concept of Large-coessential submodule as a generalization of coessential submodule, such that a submodule $K$ of an R-module $M$ is said to be Large-coessential submodule, if $\frac{N}{K} \ll_{L} \frac{M}{K^{\prime}}$, where $K \leq N \leq M$. In section one, we give many properties of this kind of submodule. In section two, we introduce the concept of Large-coclosed submodule, as a generalization of coclosed submodule, such that a submodule $N$ of an R-module $M$ is called Large-coclosed submodule, if $K$ is Large-coessential submodule of $N$ in $M$, for some submodule $K$ of $N$, implies that $K=N$. Also, we give some basic properties of this kind of submodules. We give, in Lemma(1.1), some properties of Large-small (L-small) submodule of $M$, that were introduced earlier [4] and are needed in this paper.

Lemma 1.1[4]: 1- Let $f: M \rightarrow M^{\top}$ be an epimorphism where $M$ and $M$ is an R-modules, such that $N \ll_{L} M$, then $f^{-1}(N) \ll_{L} M$.

2- Let $M$ be an R-module and $K, N$ are submodules of $M$ where $K$ is closed in $M$, such that $K \leq N \leq M$. If $N \ll_{L} M$, then $K \ll_{L} M$ and $\frac{N}{K} \ll_{L} \frac{M}{K}$.

3- Let $M$ be an R-module and $K_{1}, K_{2}$ are submodules of $M$, then $K_{1} \ll_{L} M$ and $K_{2} \ll_{L} M$ if and only if $K_{1} \oplus K_{2} \ll_{L} M$.

4- Let $M$ be an R-module and $K, N$, and $U$ are submodules of $M$, such that $K \leq N \leq U \leq$ $M$ and $K, N$ are closed submodules in $M$. Then, $\frac{U}{K} \ll_{L} \frac{M}{K}$ if and only if $\frac{U}{N} \ll_{L} \frac{M}{N}$ and $\frac{N}{K} \ll_{L} \frac{M}{K}$.

Now, we prove the following Lemma that we used in this paper.

Lemma 1.2: Let $M$ be an R-module and $K, N$ are submodules of $M$ such that $K \leq N \leq$ $M$. If $\frac{N}{K} \ll_{L} \frac{M}{K}$, then $N \ll_{L} M$.

Proof: Let $\pi: M \rightarrow \frac{M}{K}$ be a natural epimorphism and since $\frac{N}{K} \ll_{L} \frac{M}{K}$, then by Lemma(1.1), we get $N=\pi^{-1}\left(\frac{N}{K}\right) \ll_{L} M$, hence $N \ll_{L} M$.

\section{Large-Coessential submodule}

In this section we introduce the concept of Large- coessential submodule and many of its properties.

Definition 2.1: Let $M$ be an R-module and $K, N$ are submodules of $M$ such that $K \leq N \leq M$, then $K$ is called Large-coessential ( L-coessential) submodule of $N$ in $M\left(K \leq_{\text {L.ce }} N\right)$ if $\frac{N}{K} \ll_{L} \frac{M}{K}$.

\section{Remarks and Examples 2.2}

1- Every coessential submodule is L-coessential submodule.

Proof: Let $K$ be a coessential submodule of $M$ and $K \leq N$ such that $\frac{N}{K} \ll \frac{M}{K}$, then by [4], $\frac{N}{K} \ll_{L} \frac{M}{K}$ and hence $K$ is L-coessential submodule.

2- The converse of (1) is not true, as in the following example: In $Z$ as Z-module, $\{\overline{0}\}$ is Lcoessential submodule of $2 Z$ in $Z$, since $\frac{2 Z}{\{\overline{0}\}} \simeq 2 Z \ll_{L} \frac{Z}{\{\overline{0}\}} \simeq Z$. But $2 Z$ is not small in $Z$ by [4] , so $\{\overline{0}\}$ is not coessential submodule of $2 Z$.

3- In $Z_{4}$ as Z-module, $\{\overline{0}\}$ is L-coessential submodule of $\{\overline{0}, \overline{2}\}$ in $Z_{4}$, since $\frac{\{\overline{0}, \overline{2}\}}{\{\overline{0}\}} \simeq$ $\{\overline{0}, \overline{2}\} \ll_{L} \frac{Z_{4}}{\{\overline{0}\}} \simeq Z_{4}$ and since $\{\overline{0}, \overline{2}\}+Z_{4}=Z_{4}$ and $Z_{4}$ is essential in $Z_{4}$.

4- In $Z$ as Z-module, $4 Z$ is L-coessential submodule of $2 Z$ in $Z$, since $\frac{2 Z}{4 Z} \bumpeq\{\overline{0}, \overline{2}\} \ll_{L} \frac{Z}{4 Z} \bumpeq$ $Z_{4}$ by (3). 
5- In $Z_{6}$ as Z-module, $\{\overline{0}\}$ is not L-coessential submodule of $\{\overline{0}, \overline{3}\}$ in $Z_{6}$, since $\frac{\{\overline{0}, \overline{3}\}}{\{\overline{0}\}} \simeq$ $\overline{\{0}, \overline{3}\}$ and $\frac{Z_{6}}{\{\overline{0}\}} \simeq Z_{6}$ and hence $\{\overline{0}, \overline{3}\}$ is not L-small in $Z_{6}$ by [4].

6- In $Z_{8}$ as Z-module, $\{\overline{0}, \overline{4}\}$ is L-coessential submodule of $\{\overline{0}, \overline{2}, \overline{4}, \overline{6}\}$ in $Z_{8}$, since $\frac{\{\overline{0}, \overline{2}, \overline{4}, \overline{6}\}}{\{\overline{0}, \overline{4}\}} \simeq\{\overline{0}, \overline{4}\} \ll_{L} \frac{Z_{8}}{\{\overline{0}, \overline{4}\}} \simeq\{\overline{0}, \overline{2}, \overline{4}, \overline{6}\}, \quad$ since $\quad\{\overline{0}, \overline{4}\}+\{\overline{0}, \overline{2}, \overline{4}, \overline{6}\}=\{\overline{0}, \overline{2}, \overline{4}, \overline{6}\} \quad$ and $\{\overline{0}, \overline{2}, \overline{4}, \overline{6}\}$ is essential in $\{\overline{0}, \overline{2}, \overline{4}, \overline{6}\}$.

7- Let $M$ be an R-module and $K, N$ are submodules of $M$ such that $K \leq N \leq M$. If $\frac{M}{K}$ is semisimple module, then $K$ is coessential submodule of $N$ in $M$ if and only if $K$ is Lcoessential submodule of $N$ in $M$.

Proposition 2.3: Let $M$ be an R-module and $N$ be a submodule of $M$, then $N \ll_{L} M$ if and only if $\{\overline{0}\} \leq_{L . c e} N$ in $M$.

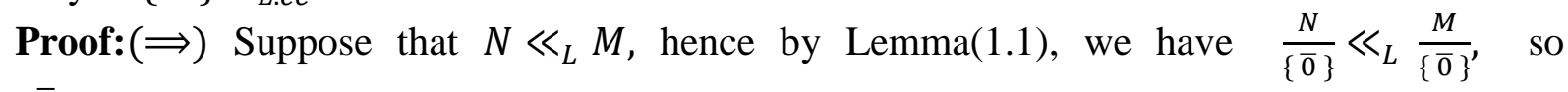
$\{\overline{0}\} \leq_{\text {L.ce }} N$ in $M$.

$(\Leftarrow)$ Let $\{\overline{0}\} \leq_{L . c e} N$ in $M$ and let $N+K=M$, where $K$ is submodule of $M$, so $\frac{N+K}{\{\overline{0}\}}=\frac{M}{\{\overline{0}\}}$, hence $\frac{N}{\{\overline{0}\}}+\frac{K}{\{\overline{0}\}}=\frac{M}{\{\overline{0}\}}$. Also, since $\{\overline{0}\} \leq_{L . c e} N$ in $M$, then $\frac{N}{\{\overline{0}\}} \ll_{L} \frac{M}{\{\overline{0}\}}$, so $\frac{K}{\{\overline{0}\}} \leq_{e} \frac{M}{\{\overline{0}\}}$, hence $K \leq_{e} M$ and then $N \ll_{L} M$.

Theorem 2.4: Let $M$ be an R-module and $K, N, U$ are submodules of $M$ such that $K \leq N \leq$ $U \leq M$ and $K$ is closed in $M$, then $K \leq_{L . c e} U$ in $M$ if and only if $U+N=M$, implies that $N \leq_{e} M$.

Proof: $(\Longrightarrow)$ Let $K \leq_{L . c e} U$ in $M$ and $\frac{U}{K}+\frac{N}{K}=\frac{M}{K}$. Since $\frac{U}{K} \ll_{L} \frac{M}{K}$, hence by Lemma(1.2), we have $U \ll_{L} M$ and $U+N=M$, so $N \leq_{e} M$.

$(\Leftarrow)$ Let $U+N=M$, so $\frac{U}{K}+\frac{N}{K}=\frac{M}{K}$. Since $N \leq_{e} M$ and $K$ is closed in $M$, then we have $\frac{N}{K} \leq_{e} \frac{M}{K}[2]$, hence $\frac{U}{K} \ll_{L} \frac{M}{K}$ and we get $K \leq_{L . c e} U$ in $M$.

Proposition 2.5: Let $M$ be an R-module and $K, N$, and $U$ are submodules of $M$ such that $K \leq N \leq U \leq M$, then $N \leq_{L . c e} U$ in $M$ if and only if $\frac{N}{K} \leq_{L . c e} \frac{U}{K}$ in $\frac{M}{K}$.

Proof: $(\Longrightarrow)$ Suppose that $N \leq_{L . c e} U$ in $M$, hence $\frac{U}{N} \ll_{L} \frac{M}{N}$. Since $\frac{U}{N} \simeq \frac{U / K}{N / K}$ and $\frac{M}{N} \simeq \frac{M / K}{N / K}$ by the Third isomorphism Theorem, then $\frac{U / K}{N / K} \ll_{L} \frac{M / K}{N / K}$ and hence $\frac{N}{K} \leq_{L . c e} \frac{U}{K}$ in $\frac{M}{K}$.

$(\Leftarrow)$ Supppose that $\frac{N}{K} \leq_{L . c e} \frac{U}{K}$ in $\frac{M}{K}$, hence $\frac{U / K}{N / K} \ll_{L} \frac{M / K}{N / K}$ and by using the Third isomorphism Theorem, we get $\frac{U}{N} \simeq \frac{U / K}{N / K} \ll_{L} \frac{M / K}{N / K} \simeq \frac{M}{N}$, hence $\frac{U}{N} \ll_{L} \frac{M}{N}$, then $N \leq_{L . c e} U$ in $M$.

Proposition 2.6: Let $M$ be an R-module and $K, N$, and $U$ are submodules of $M$, such that $K \leq N \leq U \leq M$ and $K, N$ are closed in $M$, then $K \leq_{L . c e} U$ in $M$ if and only if $K \leq_{\text {L.ce }} N$ in $M$ and $N \leq_{\text {L.ce }} U$ in $M$.

Proof: $(\Longrightarrow)$ Suppose that $K \leq_{L . c e} U$ in $M$, then $\frac{U}{K} \ll_{L} \frac{M}{K}$ and by Lemma(1.1), we have $\frac{N}{K} \ll_{L} \frac{M}{K}$ and $\frac{U}{N} \ll_{L} \frac{M}{N}$, hence $K \leq_{L . c e} N$ in $M$ and $N \leq_{L . c e} U$ in $M$.

$(\Longleftarrow)$ Supppose that $K \leq_{L . c e} N$ in $M$ and $N \leq_{L . c e} U$ in $M$, hence $\frac{N}{K} \ll_{L} \frac{M}{K}$ and $\frac{U}{N} \ll_{L} \frac{M}{N}$ and by Lemma(1.1), we get the result.

Proposition 2.7: Let $M$ be an R-module and $K, N, U$, and $H$ are submodules of $M$, such that $K \leq N \leq U \leq H \leq M$ and $K+U$ is closed in $M$. If $K \leq_{L . c e} N$ in $M$ and $U \leq_{L . c e} H$ in $M$, then $K+U \leq_{L . c e} N+H$ in $M$. 
Proof: Suppose that $K \leq_{L . c e} N$ in $M$ and $U \leq_{L . c e} H$ in $M$, hence $\frac{N}{K} \ll_{L} \frac{M}{K}$ and $\frac{H}{U} \ll_{L} \frac{M}{U}$. Thus we have $N \ll_{L} M$ and $H \ll_{L} M$ by Lemma(1.2), hence $N+H \ll_{L} M$ and $K+U$ is closed in $M$. Thus we have, $\frac{N+H}{K+U} \ll_{L} \frac{M}{K+U}$ by Lemma(1.1), hence $K+U \leq_{L . c e} N+H$ in $M$.

Corollary 2.8: Let $M$ be an R-module and $K, N$, and $U$ are submodules of $M$ such that $K \leq$ $N \leq U \leq M$. If $K \leq_{L . c e} N$ in $M$, then $K+U \leq_{L . c e} N+U$ in $M$.

Proof: Let $U \leq M$, since $U \leq_{L . c e} U$ in $M$ and $K \leq_{L . c e} N$ in $M$, then by proposition(2.7), we get $K+U \leq_{L . c e} N+U$ in $M$.

Proposition 2.9: Let $M$ be an R-module and $K, N$, and $U$ are submodules of $M$, such that $K \leq N \leq U \leq M$ and $K$ is closed in $M$. If $K \leq_{L . c e} N$ in $M$ and $U \ll_{L} M$, then $K \leq_{\text {L.ce }} N \oplus U$.

Proof: Let $\frac{H}{K}$ be a submodule of $\frac{M}{K}$ such that $\frac{N+U}{K}+\frac{H}{K}=\frac{M}{K}$. Hence, $\frac{N}{K}+\frac{U}{K}+\frac{H}{K}=\frac{M}{K}$, so we get $N+U+H=M$. Since $U \ll_{L} M$, then $N+H \leq_{e} M$ and since $K$ is closed in $M$, hence $\frac{N+H}{K} \leq_{e} \frac{M}{K}$ by [2]. Also, since $K \leq_{L . c e} N$ in $M$, then $\frac{N}{K} \ll_{L} \frac{M}{K}$, hence we get $\frac{U}{K}+\frac{H}{K}=$ $\frac{U+H}{K} \leq_{e} \frac{M}{K}$. Therefore, $\left(\frac{N+H}{K}\right) \cap\left(\frac{U+H}{K}\right) \leq_{e} \frac{M}{K}$ by [1], hence $\frac{(N+H) \cap(U+H)}{K} \leq_{e} \frac{M}{K}$ and then $\frac{(N \cap U)+H}{K} \leq_{e} \frac{M}{K}$. Hence, we get $\frac{H}{K} \leq_{e} \frac{M}{K}$, so $\frac{N \oplus U}{K} \ll_{L} \frac{M}{K}$, hence $K \leq_{L . c e} N \oplus U$.

Proposition 2.10: Let $M$ be an R-module and $K, N$, and $U$ are submodules of $M$ such that $K \leq N \leq U \leq M$ and $K$ is closed in $M$. If $N=K+U$ and $U \ll_{L} M$, then $K \leq_{L . c e} N$ in M.

Proof: Let $\frac{H}{K}$ be a submodule of $\frac{M}{K}$ such that $\frac{N}{K}+\frac{H}{K}=\frac{M}{K}$, hence $N+H=M$. Also, since $N=K+U$, so $M=N+H=(K+U)+H=U+H$, hence $M=U+H$. Since $U \ll_{L} M$, we get $H \leq_{e} M$ and $K$ is closed in $M$, then $\frac{H}{K} \leq_{e} \frac{M}{K}$ by [2], hence $\frac{N}{K} \ll_{L} \frac{M}{K}$, so $K \leq_{L . c e} N$ in $M$.

Proposition 2.11: Let $f: M \rightarrow N$ be an epimorphism where $M$ and $N$ are R-modules. If $K \leq_{L . c e} U$ in $N$ such that $f^{-1}(K)$ is closed in $M$,then $f^{-1}(K) \leq_{L . c e} f^{-1}(U)$ in $M$.

Proof: Let $\frac{H}{f^{-1}(K)}$ be a submodule of $\frac{M}{f^{-1}(K)}$ such that $\frac{f^{-1}(U)}{f^{-1}(K)}+\frac{H}{f^{-1}(K)}=\frac{M}{f^{-1}(K)}$, so $f^{-1}(U)+$ $H=M$ and hence $U+f(H)=N$, so $\frac{U}{K}+\frac{f(H)}{K}=\frac{N}{K}$. Also, since $K \leq_{L . c e} U$ in $N$, then $\frac{U}{K} \ll_{L} \frac{N}{K}$ and hence $\frac{f(H)}{K} \leq_{e} \frac{N}{K}$, then $f(H) \leq_{e} N$. Thus, $H=f^{-1}(f(H)) \leq_{e} M$. Since $f^{-1}(K)$ is closed in $M$, then $\frac{H}{f^{-1}(K)} \leq_{e} \frac{M}{f^{-1}(K)}$ by [2], so $\frac{f^{-1}(U)}{f^{-1}(K)} \ll_{L} \frac{M}{f^{-1}(K)} \quad$ and hence $f^{-1}(K) \leq_{L . c e} f^{-1}(U)$ in $M$.

Proposition 2.12: Let $M$ be an R-module and $K, N$, and $U$ are submodules of $M$, then the followings are equivalent:

1- If $K \leq_{\text {L.ce }} K+N$, then $K \cap N \leq_{\text {L.ce }} N$.

2- If $K \leq_{\text {L.ce }} N$ and $V \leq M$, then $K \cap V \leq_{\text {L.ce }} N \cap V$.

3- If $K \leq_{\text {L.ce }} N$ and $W \leq_{L . c e} U$, then $K \cap W \leq_{L . c e} N \cap U$.

Proof: $(1) \Rightarrow(2)$ Let $K \leq_{L . c e} N$ and $V \leq M$. Since $K+(N \cap V) \leq N$, then by proposition(2.6), we get $K \leq_{L . c e} K+(N \cap V)$ in $M$. Hence from (1), $K \cap(N \cap$ $V) \leq_{L . c e} N \cap V$ in $M$, so $K \cap V \leq_{L . c e} N \cap V$.

(2) $\Rightarrow$ (3) Let $K \leq_{L . c e} N$ in $M$ and $W \leq M$, hence from (2), $K \cap W \leq_{\text {L.ce }} N \cap W$. Also, $W \leq_{L . c e} U$ and $N \leq M$, hence from(2), $N \cap W \leq_{L . c e} N \cap U$, then by proposition(2.6), we get $K \cap W \leq_{\text {L.ce }} N \cap U$.

(3) $\Rightarrow$ (1) Let $K \leq_{\text {L.ce }} K+N$. Since $N \leq_{\text {L.ce }} N$, then from (3) we get $K \cap N \leq_{\text {L.ce }}(K+$ $N) \cap N$ and hence, $K \cap N \leq_{\text {L.ce }} N$. 


\section{Large-Coclosed submodule}

In this section we introduce the concept of Large-coclosed submodule and some of its properties.

Definition 3.1: Let $M$ be an R-module and $N$ be a submodule of $M$, then $N$ is called Largecoclosed (L-coclosed) submodule of $M\left(N \leq_{L . c c} M\right)$ if $K \leq_{L . c e} N$ in $M$ for some submodule $K$ of $N$, implies that $K=N$. Equivalently, $N$ is called Large-coclosed (L-coclosed) submodule of $M$, if $N$ has no proper L-coessential submodule of $M$.

Let $N, K$ be submodules of $M$ such that $N \leq K \leq M$, then $N$ is called Large-coclosure (L-coclosure) submodule of $K$ in $M$, if $N \leq_{\text {L.ce }} K$ in $M$ and $N \leq_{L . c c} M$.

\section{Remarks and Examples 3.2}

1- Every L-coclosed submodule is coclosed submodule.

Proof: Let $N$ be L-coclosed submodule of $M$ and $K \leq N$, such that $\frac{N}{K} \ll \frac{M}{K}$. Hence by [4], $\frac{N}{K} \ll_{L} \frac{M}{K}$, so $K \leq_{L . c e} N$ in $M$. Since $N$ is L-coclosed of $M$, then $K=N$ and hence $N$ is coclosed submodule.

2- The converse of (1) is not true, as in the following example: In $Z_{6}$ as Z-module: $\{\overline{0}, \overline{2}, \overline{4}\}$ is coclosed submodule of $Z_{6}$, since $\{\overline{0}\}$ is the only submodule of $\{\overline{0}, \overline{2}, \overline{4}\}$, such that $\frac{\{\overline{0}, \overline{2}, \overline{4}\}}{\{\overline{0}\}} \simeq$ $\{\overline{0}, \overline{2}, \overline{4}\}, \frac{Z_{6}}{\{\overline{0}\}} \simeq Z_{6}$, and $\{\overline{0}, \overline{2}, \overline{4}\}$ is not small in $Z_{6}$. Also, $\{\overline{0}\} \neq\{\overline{0}, \overline{2}, \overline{4}\}$, but $\{\overline{0}, \overline{2}, \overline{4}\}$ is not L-coclosed, since $\{\overline{0}, \overline{2}, \overline{4}\}$ is L-small in $Z_{6}$, but $\{\overline{0}\} \neq\{\overline{0}, \overline{2}, \overline{4}\}$.

3- In $Z_{6}$ as Z-module: $\{\overline{0}, \overline{3}\}$ is L-coclosed of $Z_{6}$, since $\{\overline{0}\}$ is not L-coessential submodule of $\{\overline{0}, \overline{3}\}$, by $(2.2)$, and $\{\overline{0}\} \neq\{\overline{0}, \overline{3}\}$.

4- In $Z_{4}$ as Z-module: $\{\overline{0}, \overline{2}\}$ is not L-coclosed of $Z_{4}$, since $\{\overline{0}\} \leq_{L . c e}\{\overline{0}, \overline{2}\}$ by (2.2), but $\{\overline{0}\} \neq\{\overline{0}, \overline{2}\}$.

5- In $Z_{8}$ as Z-module: $\{\overline{0}, \overline{2}, \overline{4}, \overline{6}\}$ in not L-coclosed of $Z_{8}$, since $\{\overline{0}, \overline{4}\} \leq_{\text {L.ce }}\{\overline{0}, \overline{2}, \overline{4}, \overline{6}\}$ by (2.2), but $\{\overline{0}, \overline{4}\} \neq\{\overline{0}, \overline{2}, \overline{4}, \overline{6}\}$.

6- In Z as Z-module: $2 \mathrm{Z}$ is not L-coclosed of $\mathrm{Z}$, since $4 \mathrm{Z} \leq_{L . c e} 2 \mathrm{Z}$ by (2.2), but $4 \mathrm{Z} \neq 2 \mathrm{Z}$.

7- Let $M$ be an R-module and $K, N$ are submodules of $M$ such that $K \leq N \leq M$. If $\frac{M}{K}$ is semisimple module, then $N$ is coclosed submodule of $M$ if and only if $N$ is L-coclosed submodule .

Proposition 3.3: Let $M$ be an R-module and $K, U$, and $N$ are submodules of $M$ such that $U \leq K \leq N \leq M$, then $N \leq_{L . c C} M$ if and only if $\frac{N}{U} \leq_{L . c c} \frac{M}{U}$.

Proof: $\Rightarrow$ ) let $\frac{K}{U} \leq \frac{N}{U}$ and $\frac{K}{U} \leq_{L . c e} \frac{N}{U}$ in $\frac{M}{U}$, so by proposition(2.5) we get $K \leq_{L . c e} N$ in $M$. Since $N \leq_{L . c C} M$, then $K=N$ and hence $\frac{K}{U}=\frac{N}{U}$.

$(\Leftarrow)$ Let $K \leq_{L . c e} N$ in $M$, so by proposition(2.5) we get $\frac{K}{U} \leq_{L . c e} \frac{N}{U}$ in $\frac{M}{U}$. Since $\frac{N}{U} \leq_{L . c c} \frac{M}{U}$, then $\frac{K}{U}=\frac{N}{U}$ and hence $K=N$.

Proposition 3.4: Let $M$ be an R-module and $N$ be a nonzero submodule of $M$, then either $N \ll_{L} M$ or $N \leq_{L . c c} M$, but not both.

Proof: Suppose that $N$ be a nonzero submodule of $M$ and let $N$ be not L-coclosed submodule.

Then, there exists a proper submodule $K$ of $N$ such that $K \leq_{L . c e} N$, hence $\frac{N}{K} \ll_{L} \frac{M}{K}$ and by Lemma(1.2), we get $N \ll_{L} M$. Now, if $N \leq_{L . c c} M$ and by supposing that $N \ll_{L} M$, let $\{\overline{0}\} \leq N$ such that $\{\overline{0}\} \leq_{L . c e} N$, so $\frac{N}{\{\overline{0}\}} \ll_{L} \frac{M}{\{\overline{0}\}}$. Since $N \leq_{L . c c} M$, then $\{\overline{0}\}=N$, but this is a contradiction, hence $N$ is not L-small in $M$.

Lemma 3.5: Let $M$ be an R-module and $U, K$, and $N$ are submodules of $M$ such that $U \leq K \leq$ $N \leq M$. If $\frac{K}{\mathrm{U}} \ll_{L} \frac{N}{\mathrm{U}}$ and $\frac{N}{\mathrm{U}} \ll_{L} \frac{M}{\mathrm{U}}$, then $\frac{K}{\mathrm{U}} \ll_{L} \frac{M}{\mathrm{U}}$. 
Proof: Let $\frac{H}{\mathrm{U}}$ be a submodule of $\frac{M}{\mathrm{U}}$ such that $\frac{K}{\mathrm{U}}+\frac{H}{\mathrm{U}}=\frac{M}{\mathrm{U}}$. Hence $K+H=M$, so $N+H=M$, hence $\frac{N}{\mathrm{U}}+\frac{H}{\mathrm{U}}=\frac{M}{\mathrm{U}}$. Since $\frac{N}{\mathrm{U}} \ll_{L} \frac{M}{\mathrm{U}}$, then $\frac{H}{\mathrm{U}} \leq_{e} \frac{M}{\mathrm{U}}$ and hence $\frac{K}{U} \ll_{L} \frac{M}{\mathrm{U}}$.

Proposition 3.6: Let $M$ be an R-module and $U, K$, and $N$ are submodules of $M$ such that $U \leq K \leq N \leq M$. If $K \leq_{L . c c} M$ and $\frac{N}{\mathrm{U}} \ll_{L} \frac{M}{\mathrm{U}^{\prime}}$ then $K \leq_{L . c c} N$.

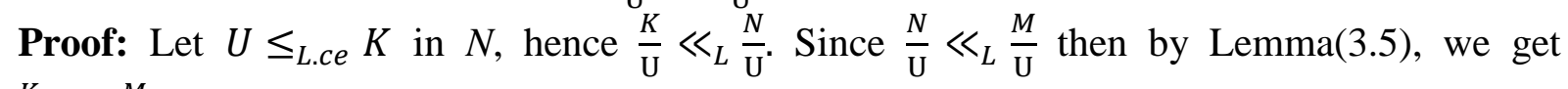
$\frac{K}{\mathrm{U}} \ll_{L} \frac{M}{\mathrm{U}}$, hence $U \leq_{L . c e} K$ in $M$. Also, since $K \leq_{L . c c} M$, then $U=K$, so $K \leq_{L . c c} N$.

\section{References}

[1] Kasch, F. Modules and Rings. Academic Press: Inc-London, 1982.

[2] Goodearl, K. R. Ring Theory: Nonsingular Rings and Modules. Marcel Dekkl, 1976.

[3] Ganesan, L. and Vanaja, N. "Modules for which every submodule has a unique coclosure". Comm. Algebra, vol.30, no.5, pp. 2355-2377, 2002.

[4] Amira A. A. and Sahira M. Y. " On Large-Small submodule and Large-Hollow module". Journal of Physics: Conference Series, vol. 1818, 2021.

[5] Firas Sh. F and Sahira M. Y. "ET-Coessential and ET-Coclosed submodules". Iraqi Journal of Science, vol.60, no.12, pp. 2706-2710, 2019.

[6] Omar K. I. and Alaa A. E. "R-annihilator-Coessential and R-annihilator-Coclosed Submodules". Iraqi Journal of Science, vol.61, no.4, pp.820-823, 2020.

[7] Hassan S. 2017. On (G*-)T-lifting Modules and T-H supplemented Modules. M.Sc. Thesis, Department of Mathematics, College of Science, Baghdad University, Iraq.

[8] Enas M. K. and Wasan Kh. "On generalization of small submodule". Sci.Int.(Lahore), vol.30, no.3, pp.359-365, 2018. 